\title{
OBTENÇÃO DE ISOTERMAS DE DESSORÇÃO DE COGUMELO in natura E DESIDRATADO OSMOTICAMENTE ${ }^{1}$
}

\author{
Louise E. KUROZAWA2,*, Ânoar A. EL-AOUAR², Fernanda E. X. MURR²
}

\begin{abstract}
RESUMO
O presente trabalho teve como objetivo a obtenção de isotermas de dessorção de cogumelo Agaricus blazei nas formas in natura e desidratada osmoticamente com solução de $\mathrm{NaCl}(10 \% \mathrm{p} / \mathrm{p})$ a $20^{\circ} \mathrm{C}$. As isotermas foram determinadas nas temperaturas de 40,60 e $80^{\circ} \mathrm{C}$, através do método gravimétrico estático com soluções salinas saturadas. Os dados experimentais foram ajustados a cinco modelos da literatura (BET, GAB, Halsey, Henderson e Oswin) e a dois modelos com o parâmetro temperatura (Henderson modificado e Chung-Pfost). O desvio relativo médio entre os valores experimentais e os valores preditos foi calculado para cada curva para se avaliar o modelo que apresentou melhor ajuste. Assim, observou-se que os modelos de Henderson e Henderson modificado foram os que melhor se ajustaram dentre as equações sem e com o parâmetro temperatura tanto para o cogumelo in natura quanto para o desidratado osmoticamente. Palavras-chave: isotermas de dessorção, desidratação osmótica, modelagem matemática, atividade de água, cogumelo Agaricus blazei.
\end{abstract}

\section{SUMMARY}

SORPTION ISOTHERMS FOR FRESH AND OSMOTICALLY DEHYDRATED MUSHROOMS. The present paper aimed at obtaining the desorption isotherms of Agaricus blazei mushroom in nature, which suffered osmotic dehydration. The osmotic treatment was carried out at $20^{\circ} \mathrm{C}$ with $10 \% \mathrm{w} / \mathrm{w}$ salt solution. The isotherms were obtained for three different temperatures $\left(40,60\right.$ and $\left.80^{\circ} \mathrm{C}\right)$ using the static method with saturated salt solutions. Experimental data were fitted by five models (BET, GAB, Halsey, Henderson and Oswin) and two models with the temperature as an independent variable (modified Henderson and Chung-Pfost). The average relative deviations between experimental and predicted data were obtained to choose the best model. The best fitting was obtained when the traditional and modified Henderson models were used.

Keywords: desorption isotherms, osmotic dehydration, mathematical modeling, water activity, Agaricus blazei mushroom.

\section{1 - INTRODUÇÃO}

O Agaricus blazei é um cogumelo comestível, conhecido popularmente no Brasil como Cogumelo do Sol, Cogumelo de Deus ou Cogumelo Piedade. É oriundo das regiões serranas da Mata Atlântica do sul do estado de São Paulo e cultivado em outros países, tais como Japão, China e Indonésia. No Brasil, o cogumelo Agaricus blazei é cultivado principalmente nos estados de São Paulo, Minas Gerais e Paraná [4].

Popularmente, é utilizado no combate ao estresse físico e psicológico, para estimular a imunidade, melhorar a qualidade de vida dos diabéticos, reduzir colesterol, combater doenças como osteoporose e úlcera gástrica e aliviar problemas digestivos. É usado também como antioxidante e anticarcinogênico [14]. Somente nos últimos anos, com o desenvolvimento de técnicas mais precisas de isolamento e purificação de substâncias químicas, é que foi possível comprovar cientificamente a ação terapêutica de alguns cogumelos, isolando-se substâncias tanto de ação antitumoral quanto bacteriana [6].

Os cogumelos possuem um teor de umidade ao redor de $90 \%$ e são altamente perecíveis. Assim, há uma necessidade de processar o produto, de modo a aumentar sua vida de prateleira [20]. Um processo que vem sendo bastante utilizado para tal finalidade é a desidratação osmótica, na qual ocorre uma diminuição da atividade de água do produto, contribuindo para sua conservação e, conseqüentemente, o aumento da vida de prateleira do mesmo, devido à redução no crescimento microbiano e nas reações bioquímicas que dependem da umidade do produto.

${ }^{1}$ Recebido para publicação em 23/06/2005. Aceito para publicação em 12/10/2005 (001555).

${ }^{2}$ Universidade Estadual de Campinas - Departamento de Engenharia de Alimentos - Caixa Postal 6121 - CEP: 13083-970 - Campinas - SP - Brasil - Telefone: (19) 3788-4057.

*A quem a correspondência deve ser enviada: louisek98@yahoo.com.br.
O processo de desidratação osmótica baseia-se na imersão de alimentos, inteiros ou em pedaços, em soluções hipertônicas de um ou mais solutos, originando dois fluxos simultâneos e em contracorrente: uma saída de água do produto para a solução e uma migração de solutos da solução para o alimento. Há um terceiro fluxo envolvido no processo, que consiste na perda de alguns sólidos naturais, como açúcares, minerais, entre outros nutrientes. Embora seja insignificante em relação aos outros dois fluxos principais, pode ser importante para as qualidades organolépticas e nutricionais do produto [17].

Ao se expor um material biológico a uma determinada umidade relativa, ocorre um fenômeno de transferência de massa para se alcançar um equilíbrio dinâmico entre a umidade do produto e a do ambiente. Tal fato acontece quando a pressão de vapor da água na superfície do material se iguala à do ar que o envolve [22].

Assim, no equilíbrio, a atividade de água $\left(a_{w}\right)$ está relacionada com a umidade relativa (\%UR) do ambiente através da seguinte equação:

$$
a_{w}=\frac{P_{w}}{P_{v}}=\frac{U R}{100}
$$

Onde: $P_{w}=$ pressão parcial da água contida no alimento; $P_{v}=$ pressão de vapor da água pura à mesma temperatura.

O estudo da atividade de água pode ser feito através das isotermas de sorção. Uma isoterma é uma curva que descreve a relação de equilíbrio entre o teor total de umidade de um alimento e a pressão de vapor ou umidade relativa, a uma dada temperatura. Essa relação depende de diversos fatores, tais como da estrutura física da superfície do material, composição química e afinidade com a água. Portanto, como 
cada alimento apresenta características distintas de sorção de umidade, faz-se necessária a determinação experimental das curvas de sorção para cada tipo de produto [5].

Isotermas de sorção são importantes na análise e controle de vários processos na indústria de alimentos, tais como preservação, secagem, definição de embalagem e misturas. As isotermas de sorção podem ser usadas para predizer as mudanças na estabilidade do alimento e para selecionar o material de embalagem e os ingredientes apropriados para a formulação de um produto alimentício [25].

Uma isoterma de sorção pode ser obtida em duas direções: adsorção e dessorção. A primeira é feita quando um material mais seco é colocado em várias atmosferas, aumentando a umidade relativa e medindo o aumento de peso devido ao ganho de água. Na segunda, o material inicialmente úmido é colocado sob as mesmas condições ambientais utilizadas na adsorção, sendo medida a perda de peso, devido à saída de água [13]. A isoterma de dessorção possui valores de umidade de equilíbrio superiores aos da isoterma de adsorção a uma dada atividade de água. A defasagem entre estas duas curvas, denominada histerese, pode ocorrer devido a diversos fatores, tais como condensação capilar, mudanças na estrutura física do material, impurezas na superfície e mudança de fase [18].

Existem vários modelos na literatura capazes de predizer o comportamento de sorção de diversos produtos (Tabela 1). As equações de Halsey e Oswin são utilizadas para analisar isotermas de sorção de diversos alimentos, sendo que o modelo de Halsey mostra um bom ajuste para produtos ricos em amido, tais como milho, batata e trigo. O modelo de Henderson é um dos mais empregados e descreve bem o comportamento de alimentos com uma ampla faixa de atividade de água $(0,10$ a 0,75$)$, como grãos, cereais e frutas. A equação de
BET é amplamente usada para fornecer uma estimativa da umidade na monocamada adsorvida na superfície do alimento. Entretanto, essa equação apresenta um ajuste satisfatório para atividade de água entre 0,05 e 0,45 [7]. Já o modelo de GAB permite um melhor ajuste dos dados de sorção dos alimentos até atividade de água de 0,9 [23].

Assim, o objetivo deste trabalho foi obter isotermas de dessorção de cogumelo Agaricus blazei nas formas in natura e desidratada osmoticamente com solução de $\mathrm{NaCl}$ a diferentes temperaturas.

\section{2 - MATERIAL E MÉTODOS}

Para a realização deste trabalho, foram utilizados cogumelos da espécie Agaricus blazei cultivados em estufas, fornecidos pela empresa GAPI (Grupo Agaricus de Piedade), localizada na cidade de Pilar do Sul - SP. A aparência do cogumelo e o seu conteúdo de umidade foram adotados como parâmetros de padronização da amostra, sendo utilizados os cogumelos com 90\% de umidade (b.u.), coloração amarela clara e textura firme. Não foram utilizados cogumelos que apresentaram injúrias físicas e manchas.

Para a desidratação osmótica, a matéria-prima, devidamente cortada em fatias longitudinais com aproximadamente $5 \mathrm{~mm}$ de espessura, foi colocada em béqueres com solução de $10 \%$ de sal (p/p). As amostras foram então colocadas em uma incubadora da marca TECNAL (modelo TE-421), com agitação de $80 \mathrm{rpm}$ e temperatura controlada de $20^{\circ} \mathrm{C}$. Após 60 minutos de processo, as amostras foram retiradas da solução, enxaguadas com água destilada para retirar o excesso de solução osmótica e colocadas em papel absorvente.

As umidades de equilíbrio de cogumelo in natura e desidratado osmoticamente foram determinadas por método gravimétrico estático, utilizando-se soluções salinas

TABELA 1 - Modelos da literatura para ajuste de isotermas de sorção

\begin{tabular}{|c|c|c|}
\hline Nome do modelo & Modelo* & \\
\hline BET linear [5] & $X_{e}=\frac{X_{e} C_{B E T} a_{w}}{\left(1-a_{w}\right)\left[1-\left(C_{B E T}-1\right) a_{w}\right]}$ & (2) \\
\hline Halsey [10] & $a_{w}=\exp \left[\frac{-A}{X_{e}^{B}}\right]$ & (4) \\
\hline Oswin [15] & $X_{e}=A\left(\frac{a_{w}}{1-a_{w}}\right)^{B}$ & (5) \\
\hline Henderson [11] & $1-a_{w}=\exp \left(-k X_{e}^{n^{i}}\right)$ & (6) \\
\hline Henderson modificado [11] & $1-a_{w}=\exp \left(-k T X_{e}^{n^{1}}\right)$ & (7) \\
\hline
\end{tabular}

*Onde: $A, B, C_{B E T}, C_{G A B}, K_{G A B}, k, n, n^{\prime}=$ constantes das equações; $X_{e}=$ umidade de equilíbrio expressa em base seca (g água/g massa seca); $X_{m}=$ umidade na monocamada molecular (g água/g massa seca); $R=$ constante universal dos gases (J/mol.K); $T=$ temperatura $(\mathrm{K})$. 
saturadas em água destilada, para uma determinada faixa de umidade relativa, de acordo com a Tabela 2 [9].

As amostras (em triplicata) foram colocadas em cadinhos de alumínio e, em seguida, armazenadas em potes herméticos com as respectivas soluções salinas, para cada valor de umidade relativa desejada. Os potes herméticos foram colocados em estufa (marca FANEM, modelo 320-SE), nas temperaturas de 40,60 e $80^{\circ} \mathrm{C}$. As amostras foram pesadas em balança analítica (marca BOSCH, modelo SAE 200) em intervalos regulares de tempo até o equilíbrio. Alcançado o equilíbrio, as amostras foram levadas para estufa a vácuo (marca SUPRILAB, modelo EST920), a uma temperatura de $70^{\circ} \mathrm{C}$ durante $48 \mathrm{~h}$ para se obter sua massa seca [2].

Para o ajuste das isotermas do cogumelo, foram testados diversos modelos encontrados na literatura. Os parâmetros destas equações foram determinados através de uma análise de regressão linear e não-linear dos dados experimentais, realizada pelo software Statistica 5.0 [21], usando como método de ajuste os modelos Quasi-Newton e Simplex. Os critérios utilizados para escolha do melhor ajuste foram: o coeficiente de determinação $\left(R^{2}\right)$ entre as respostas observadas e os valores preditos pelo modelo e o módulo do desvio relativo médio $(E)$, cuja definição encontra-se descrita pela Equação (9):

$$
E=\frac{100}{N} \sum_{i=1}^{N} \frac{\left|V_{P}-V_{0}\right|}{V_{0}}
$$

Onde: $E=$ desvio relativo médio (\%); $V_{P}=$ valores preditos pelo modelo; $V_{o}=$ valores observados experimentalmente; $N=$ número de pontos experimentais.

De acordo com AGUERRE, SUAREZ e VIOLLAZ (1989), valores de desvio relativo médio abaixo de $10 \%$ indicam um ajuste bom para os dados experimentais.

\section{3 - RESULTADOS E DISCUSSÃo}

A Tabela 3 relaciona os valores experimentais médios de umidade de equilíbrio $\left(X_{e}\right)$ com a atividade de água $\left(a_{w}\right)$ determinada pelas soluções salinas saturadas, para a construção das isotermas de dessorção.

Os dados experimentais das isotermas de dessorção do cogumelo foram ajustados pelos modelos descritos sem o parâmetro temperatura, obtendo-se o coeficiente de determinação $\left(R^{2}\right)$ e o desvio médio relativo $(E)$. A escolha do melhor ajuste foi feita analisando o desvio médio relativo para cada modelo e para cada condição estudada. Estes valores, juntamente com os parâmetros de ajuste das equações, encontram-se descritos nas Tabelas 4 e 5.

A partir da Tabela 4 observou-se que, dentre os modelos analisados, o modelo de Henderson foi o que apresentou melhor ajuste para o cogumelo in natura, com um desvio relativo médio inferior a $11 \%$ e coeficiente de determinação próximo à unidade. Já para o cogumelo desidratado osmoticamente (Tabela 5), observou-se também que o modelo de Henderson foi o que apresentou melhor ajuste

TABELA 2 - Valores de atividade de água relacionados às soluções salinas saturadas nas temperaturas de 40,60 e $80^{\circ} \mathrm{C}$

\begin{tabular}{lccc}
\hline \multirow{2}{*}{ Sal } & & $\mathbf{a}_{\mathbf{w}}$ & \\
\cline { 2 - 4 } & $\mathbf{4 0}{ }^{\circ} \mathbf{C}$ & $\mathbf{6 0}{ }^{\circ} \mathbf{C}$ & $\mathbf{8 0}^{\circ} \mathbf{C}$ \\
$\mathrm{LiCl}$ & 0,1121 & 0,1095 & 0,1051 \\
$\mathrm{KF}$ & 0,2268 & 0,2077 & 0,2285 \\
$\mathrm{MgCl}_{2}$ & 0,3160 & 0,2926 & 0,2605 \\
$\mathrm{NaBr}$ & 0,5317 & 0,4926 & 0,5143 \\
$\mathrm{KI}$ & 0,6609 & 0,6311 & 0,6097 \\
$\mathrm{NaNO}_{3}$ & 0,7100 & 0,6735 & 0,6522 \\
$\mathrm{NaCl}$ & 0,7468 & 0,7450 & 0,7629 \\
$\mathrm{KCl}$ & 0,8232 & 0,8025 & 0,7890 \\
\hline
\end{tabular}

TABELA 3 - Valores experimentais de umidade de equilíbrio de dessorção de cogumelo in natura (IN) e desidratado osmoticamente (DO) nas temperaturas de 40,60 e $80^{\circ} \mathrm{C}$

\begin{tabular}{|c|c|c|c|c|c|c|c|c|}
\hline \multicolumn{3}{|c|}{$40^{\circ} \mathrm{C}$} & \multicolumn{3}{|c|}{$60^{\circ} \mathrm{C}$} & \multicolumn{3}{|c|}{$80^{\circ} \mathrm{C}$} \\
\hline$a_{w}$ & $X_{e}(I N)$ & $X_{e}(D O)$ & $a_{w}$ & $X_{e}(I N)$ & $X_{e}(D O)$ & $a_{w}$ & $X_{e}(I N)$ & $X_{e}(D O)$ \\
\hline 0,1121 & 0,0039 & 0,0106 & 0,1095 & 0,0042 & 0,0048 & 0,1051 & 0,0035 & 0,0063 \\
\hline 0,3160 & 0,0579 & 0,0513 & 0,2926 & 0,0500 & 0,0340 & 0,2605 & 0,0254 & 0,0247 \\
\hline 0,5317 & 0,1370 & 0,2109 & 0,4966 & 0,1652 & 0,1682 & 0,5143 & 0,1091 & 0,1301 \\
\hline 0,7100 & 0,4143 & 0,6190 & 0,6735 & 0,3067 & 0,4720 & 0,6522 & 0,1659 & 0,3331 \\
\hline 0,7468 & 0,6241 & - & 0,745 & 0,4024 & - & 0,7629 & 0,2947 & 0,4931 \\
\hline 0,8232 & - & - & 0,8025 & 0,4705 & - & 0,7890 & 0,3805 & 0,7083 \\
\hline
\end{tabular}


TABELA 4 - Constantes de ajuste da isoterma de dessorção do cogumelo in natura dos modelos sem o parâmetro temperatura

\begin{tabular}{|c|c|c|c|c|c|c|}
\hline \multirow[t]{2}{*}{ Modelos } & \multirow[t]{2}{*}{ Temperatura } & \multicolumn{3}{|c|}{ Constantes } & \multirow[t]{2}{*}{$\mathbf{R}^{2}$} & \multirow[t]{2}{*}{$E(\%)$} \\
\hline & & $\mathbf{X}_{\mathrm{m}}$ & & $\mathrm{C}_{\mathrm{BET}}$ & & \\
\hline \multirow[t]{3}{*}{ BET linear } & $60^{\circ} \mathrm{C}$ & 0,113 & & 2,259 & 0,967 & 36,12 \\
\hline & & $X_{m}$ & $\mathrm{C}_{\mathrm{GAB}}$ & $K_{G A B}$ & & \\
\hline & $40^{\circ} \mathrm{C}$ & 0,631 & 0,088 & 1,026 & 0,993 & 29,18 \\
\hline \multirow{2}{*}{ GAB } & & A & & B & & \\
\hline & $40^{\circ} \mathrm{C}$ & 0,204 & & 0,605 & 0,990 & 16,52 \\
\hline \multirow[t]{3}{*}{ Halsey } & $60^{\circ} \mathrm{C}$ & 0,216 & & 0,530 & 0,951 & 17,40 \\
\hline & $80^{\circ} \mathrm{C}$ & 0,128 & & 0,686 & 0,958 & 20,23 \\
\hline & & k & & n' & & \\
\hline Henderson & $40^{\circ} \mathrm{C}$ & 0,106 & & 1,601 & 0,990 & 18,54 \\
\hline \multirow[t]{2}{*}{ Oswin } & $60^{\circ} \mathrm{C}$ & 0,163 & & 0,802 & 0,972 & 111,40 \\
\hline & $80^{\circ} \mathrm{C}$ & 0,084 & & 1,114 & 0,990 & 25,56 \\
\hline
\end{tabular}

Tabela 5 - Constantes de ajuste da isoterma de dessorção do cogumelo desidratado osmoticamente dos modelos sem o parâmetro temperatura

\begin{tabular}{|c|c|c|c|c|c|c|}
\hline \multirow[t]{2}{*}{ Modelos } & \multirow[t]{2}{*}{ Temperatura } & \multicolumn{3}{|c|}{ Constantes } & \multirow[t]{2}{*}{$\mathbf{R}^{2}$} & \multirow[t]{2}{*}{$E(\%)$} \\
\hline & & $\mathbf{X}_{\mathrm{m}}$ & & $\mathrm{C}_{\mathrm{BET}}$ & & \\
\hline \multirow[t]{3}{*}{ BET linear } & $60^{\circ} \mathrm{C}$ & 0,539 & & 0,118 & 0,996 & 47,47 \\
\hline & & $\mathbf{x}_{\mathrm{m}}$ & $\mathbf{C}_{\mathrm{GAB}}$ & $\mathrm{K}_{\mathrm{GAB}}$ & & \\
\hline & $40^{\circ} \mathrm{C}$ & 0,785 & 0,155 & 0,967 & 0,993 & 36,70 \\
\hline \multirow[t]{3}{*}{ GAB } & $60^{\circ} \mathrm{C}$ & 0,704 & 0,123 & 1,016 & 0,997 & 36,88 \\
\hline & & A & & B & & \\
\hline & $40^{\circ} \mathrm{C}$ & 0,308 & & 0,426 & 0,992 & 5,43 \\
\hline \multirow[t]{3}{*}{ Halsey } & $60^{\circ} \mathrm{C}$ & 0,315 & & 0,406 & 0,992 & 11,51 \\
\hline & $80^{\circ} \mathrm{C}$ & 0,228 & & 0,510 & 0,973 & 14,50 \\
\hline & & k & & n' & & \\
\hline Henderson & $40^{\circ} \mathrm{C}$ & 0,185 & & 1,388 & 0,994 & 17,89 \\
\hline \multirow[t]{2}{*}{ Oswin } & $60^{\circ} \mathrm{C}$ & 0,160 & & 1,477 & 0,983 & 16,24 \\
\hline & $80^{\circ} \mathrm{C}$ & 0,137 & & 1,203 & 0,982 & 23,37 \\
\hline
\end{tabular}

nas temperaturas de 60 e $80^{\circ} \mathrm{C}$, com um desvio relativo inferior a $10 \%$. Para a isoterma de $40^{\circ} \mathrm{C}$, apesar de o modelo de Halsey apresentar desvio relativo inferior ao valor apresentado pelo modelo de Henderson, preferiu-se utilizar este último, por ter descrito bem os dados nas temperaturas de 60 e $80^{\circ} \mathrm{C}$.

Foi feito também um ajuste dos dados experimentais a modelos da literatura que possuem o parâmetro temperatura. O que melhor se ajustou aos dados foi a equação de Henderson modificada, tanto para o cogumelo in natura quanto para o desidratado osmoticamente (Tabela 6).
As comparações entre os valores experimentais e preditos pelo modelo de Henderson e Henderson modificado para as temperaturas de 40,60 e $80^{\circ} \mathrm{C}$, se encontram descritas nas Figuras 1 e 2.

De acordo com a Figura 1, observa-se que as curvas são dependentes da temperatura, isto é, o conteúdo de umidade de equilíbrio decresce com o aumento da temperatura para valores de atividade de água superiores a 0,30. A energia cinética, associada às moléculas de água presentes nos alimentos, aumenta com o aumento da temperatura. Tal fato resulta na diminuição das forças de atração e, conse- 
Tabela 6 - Constantes de ajuste da isoterma de dessorção do cogumelo in natura e desidratado osmoticamente dos modelos com o parâmetro temperatura

\begin{tabular}{|c|c|c|c|c|c|}
\hline & \multirow[t]{2}{*}{ Modelos } & \multicolumn{2}{|c|}{ Constantes } & \multirow[t]{2}{*}{$\mathbf{R}^{2}$} & \multirow[t]{2}{*}{$E(\%)$} \\
\hline \multirow{3}{*}{ In natura } & & $k$ & $n^{\prime}$ & & \\
\hline & Henderson modificado & 0,007 & 0,593 & 0,967 & 10,42 \\
\hline & & A & B & & \\
\hline \multirow{2}{*}{$\begin{array}{l}\text { Desidratado } \\
\text { osmoticamente }\end{array}$} & & $k$ & $n^{\prime}$ & & \\
\hline & Henderson modificado & 0,005 & 0,518 & 0,988 & 7,81 \\
\hline
\end{tabular}
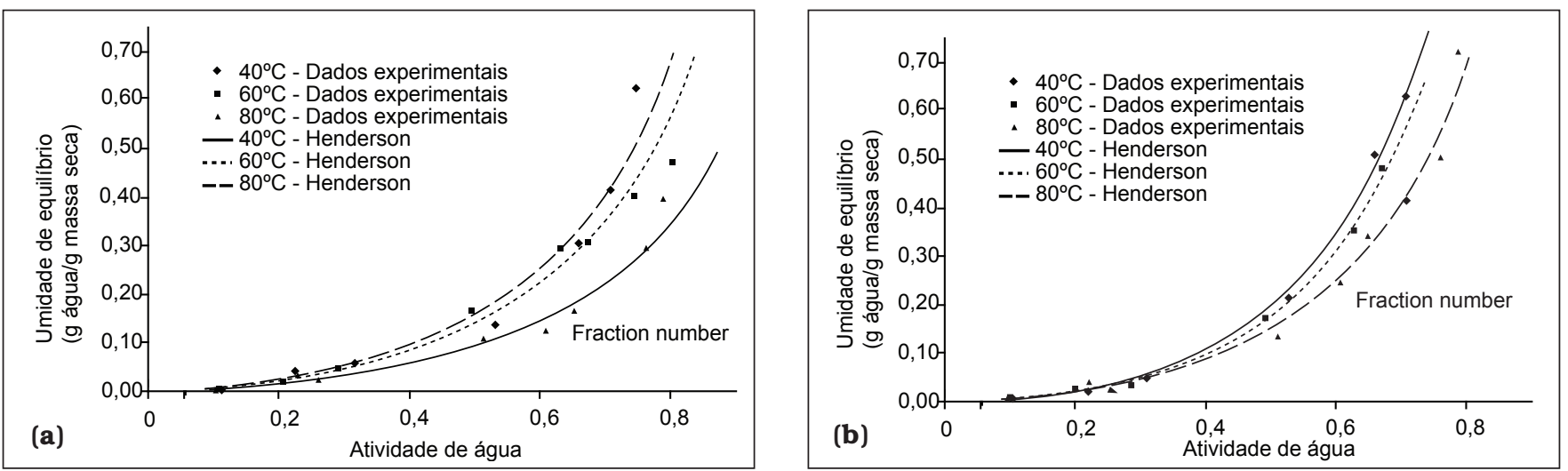

FIGURA 1 - Comparação entre os valores observados e preditos para a isoterma de dessorção do cogumelo in natura (a) e desidratado osmoticamente (b) para o modelo de Henderson (sem o parâmetro temperatura)

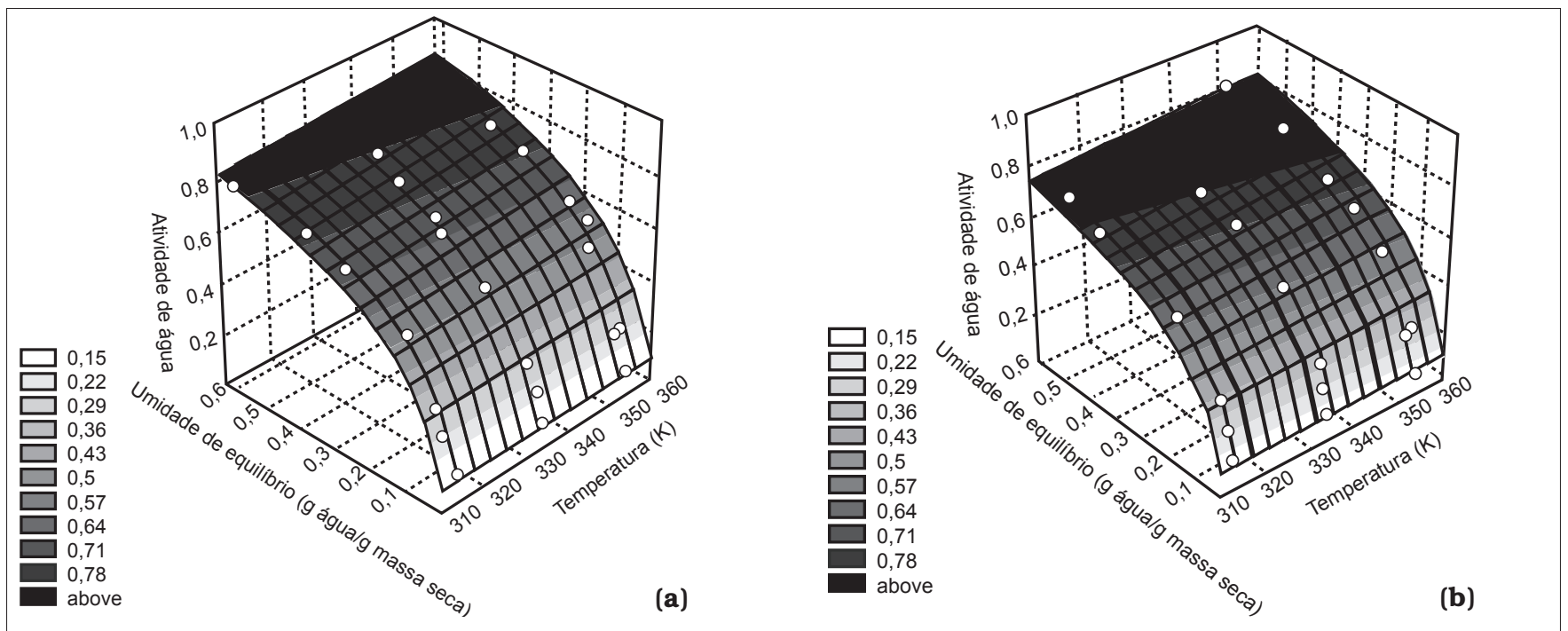

FIGURA 2 - Comparação entre os valores observados e preditos para a isoterma de dessorção do cogumelo (a) in natura e do (b) desidratado osmoticamente para o modelo de Henderson modificado (com o parâmetro temperatura)

qüentemente, na saída de água do alimento, conduzindo a um decréscimo do conteúdo de umidade com a elevação da temperatura a uma dada atividade de água [19]. SHIVHARE et al. (2004), VISWANATHAN, JAYAS e HULASARE (2003), PARK, BIN e BROD (2001) também observaram esse comportamento para diferentes alimentos.

Assim, o modelo de Henderson modificado pode ser utilizado para predizer o conteúdo de umidade de equilí- brio do cogumelo Agaricus blazei nas formas in natura e desidratado osmoticamente nas temperaturas entre $40 \mathrm{e}$ $80^{\circ} \mathrm{C}$. Este modelo é uma das equações mais empregadas e descreve bem o comportamento de muitos materiais higroscópicos, como alimentos em uma faixa de atividade de água de 0,10 a 0,75 [19]. VISWANATHAN, JAYAS e HULASARE (2003) determinaram o conteúdo de umidade de equilíbrio de fatias de cebola a temperaturas de 30, 40 
e $50^{\circ} \mathrm{C}$ e umidade relativa de 15 a $85 \%$. Os dados foram ajustados por diversos modelos da literatura, sendo o que melhor se ajustou foi o de Henderson.

Na Figura 3, foram comparados os dados experimentais das isotermas de dessorção do cogumelo in natura e desidratado osmoticamente. Nota-se que o produto pré-desidratado apresentou menores valores de atividade de água, para uma mesma umidade de equilíbrio, que o cogumelo sem tratamento, devido, provavelmente, à dissolução dos solutos impregnados. SLOAN e LABUZA (1976), citados por ISLAM e FLINK (1982), através de uma revisão bibliográfica, observaram que alguns componentes, como o sal e o glicerol, são particularmente efetivos na redução de atividade de água, ou seja, por serem substâncias eletrolíticas, dissociam-se em íons, interagindo com as moléculas de água e, conseqüentemente, diminuindo a atividade de água do produto. BARONI (2004), trabalhando com tomates tratados e não tratados osmoticamente com soluções binárias e ternárias de sal e sacarose, observou que a isoterma da fruta desidratada com solução salina apresentava menores valores de atividade de água do que o tomate in natura.

(a)
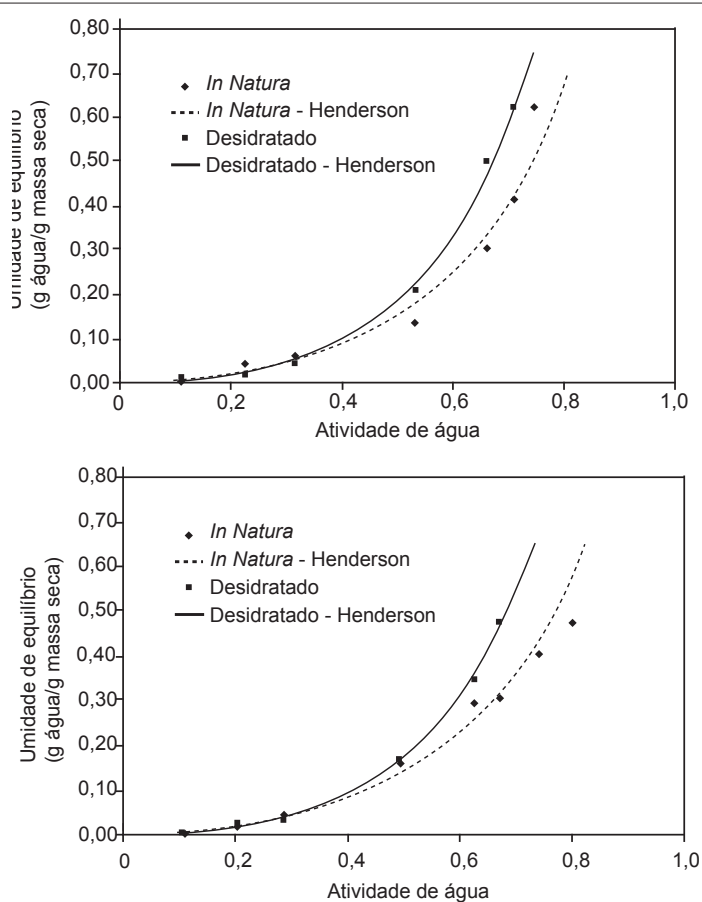

(b)

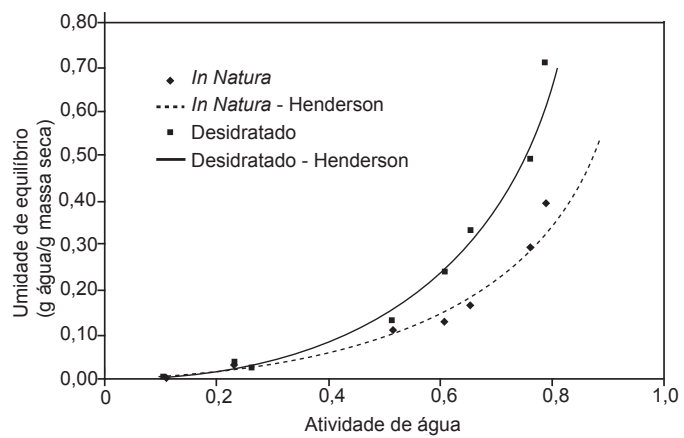

(c)

FIGURA 3. Comparação dos dados experimentais de dessorção do cogumelo in natura e desidratado osmoticamente nas temperaturas de (a) 40 , (b) 60 e (c) $80^{\circ} \mathrm{C}$

\section{4 - CONCLUSÕES}

Para o ajuste das isotermas de dessorção do cogumelo in natura aos modelos descritos sem o parâmetro temperatura, o de Henderson foi o que apresentou melhor ajuste para a temperatura de $40^{\circ} \mathrm{C}(10,54 \%), 60^{\circ} \mathrm{C}(6,66 \%)$ e $80^{\circ} \mathrm{C}$ (10,88\%). Com relação aos modelos com o parâmetro temperatura, o modelo de Henderson modificado apresentou menor valor de desvio relativo médio $(10,42 \%)$ em relação ao modelo de Chung e Pfost $(22,43 \%)$;

Para os cogumelos desidratados osmoticamente, o ajuste dos dados experimentais aos modelos sem o parâmetro temperatura, o de Henderson foi o que apresentou melhores resultados nas temperaturas de $60^{\circ} \mathrm{C}$ $(4,04 \%)$ e $80^{\circ} \mathrm{C}(7,56 \%)$, sendo que para a temperatura de $40^{\circ} \mathrm{C}$, o modelo de Halsey apresentou menor desvio relativo $(5,43 \%)$. Com relação aos modelos com o parâmetro temperatura, o de Henderson modificado foi o que apresentou melhores resultados, com um desvio relativo de $7,81 \%$;

As isotermas obtidas são dependentes da temperatura, ou seja, o conteúdo de umidade de equilíbrio decresce com o aumento da temperatura para valores de atividade de água superiores a 0,3 ;

Para as três temperaturas estudadas, as amostras tratadas osmoticamente exibiram uma clara tendência de apresentar valores de atividade de água menores do que aqueles encontrados para o cogumelo in natura.

\section{5 - REFERÊNCIAS BIBLIOGRÁFICAS}

[1] AGUERRE, R.J.; SUAREZ, C.; VIOLLAZ, P.E. New BET type multilayer sorption isotherms. Part II: Modeling water sorption in foods. Lebensm.-Wiss. Technol., v. 22, p. 192-195, 1989.

[2] AOAC. Official Methods of Analysis. 16 ${ }^{\text {th }}$ ed. Association of Official Analytical Chemists, Washington, DC, EUA, 1997.

[3] BARONI, A.F. Propriedades Mecânicas, Termodinâmicas e de Estado de Tomate Submetido à Desidratação Osmótica e Secagem. Campinas, 2004, 226 p. Tese (Doutorado em Engenharia de Alimentos), Faculdade de Engenharia de Alimentos, Universidade Estadual de Campinas (UNICAMP).

[4] BRAGA, G.C. Cogumelo do Sol: pesquisas apontam suas propriedades medicinais. Revista Tecnologia e Treinamento Agropecuário, v. 2, n. 6, p. 07, 1997.

[5] BRUNAUER, S.; EMMET, T.H.; TELLER, F. Adsorption of gases in multimolecular layers. J. Am. Chem. Soc., v. 60, n. 2, p. 309-319, 1938.

[6] CHANG, S.T.; BUSWELL, J.A. Mushroom nutriceuticals. World J. Microb. Biot., v. 12, p. 473-476, 1996.

[7] CHHINNAN, M.S.; BEUCHAT, L.R. Sorption isotherms of whole cowpeas and flours. Lebensm-Wiss. Technol., v. 18, p. 83-88, 1985.

[8] CHUNG, D.S.; PFOST, H.B. Adsorption and desorption of water vapor by cereal grains and their products. Transactions of ASAE, v. 10, p. 549, 1967.

[9] GREENSPAN, L. Humidity fixed points of binary saturated aqueous solutions. J. Res. Natı. Bureau 
Stan. - Physics and Chemistry, v. 81, n. 1, p. 8996, 1977.

[10] HALSEY, G. Physical adsorption on uniform surfaces. J. Chem. Phys., v. 16, n. 10, p. 931-937, 1948.

[11] HENDERSON, S.M. A basic concept of equilibrium moisture. Agr. Eng., St. Joseph, Michigan, v. 33, n. 1, p. 29-32, 1952.

[12] ISLAM, M.N.; FLINK, L.N. Dehydration of potato II - Osmotic concentration and its effect on air drying behavior. J. Food Tech., v. 17, n. 3, p. 387-403, 1982.

[13] LABUZA, T.P. Sorption phenomena in foods. Food Tech., v. 22, p. 263-272, 1968.

[14] MENOLI, R.C.R.N.; MANTOVANI, M.S.; RIBEIRO, L.R.; SPEIT, G.; JORDÃO, B.Q. Antimutagenic effects of the mushroom Agaricus blazei Murril extracts on V79 cells. Genet. Toxicol. Environ. Mutagenesis, v. 496, n. 1-2, p. 5-13, 2001.

[15] SWIN, C.R. The kinetics of package life. The isotherm. J. Chem. Industry (London), n. 65, p. 419-421, 1946

[16] PARK, K.J.; BIN, A.; BROD, F.P.R. Obtenção das isotermas de sorção e modelagem matemática para a pêra bartlett (Pyrus sp.) com e sem desidratação osmótica. Ciência e Tecnologia de Alimentos, v. 21. n. 1, p. 73-77, 2001.

[17] RAOULT-WACK, A.L. Recent advances in the osmotic dehydration of foods. Trends Food Sci. Tech., v. 5, n. 8, p. 255-260, 1994.

[18] RAHMAN, M.S. Food Properties Handbook. $1^{\text {st }}$ ed. CRC Press, Boca Raton, 1995.
[19] SHIVHARE, U.S.; ARORA, S.; AHMED, J.; RAGHAVAN, G.S.V. Moisture adsorption isotherms for mushroom. Lebensm-Wiss. Technol., v. 37, p. 133-137, 2004.

[20] SUGUNA, S.; USHA, M.; SREENARAYANAN, V.V.; RAGHUPATHY, R.; GOTHANDAPANI, L. Dehydration of mushroom by sun-drying, thin-layer drying, fluidized bed drying and solar cabinet drying. J. Food Sc. Tech., v. 32, n. 4, p. 284-288, 1995.

[21] Statistica for Windows 5.0. Computer program manual. Tulsa, OK: StatSoft, Inc., 1995.

[22] TREYBAL, R.E. Mass Transfer Operations. $3^{\text {rd }}$ ed. Auckland: McGraw-Hill, 1981.

[23] VAN DER BERG, C. Description of water activity of foods for engineering purposes by means of the GAB model of sorption. In: Engineering and Food. London: B. M. Mckenna, Elsevier Applied Science, v. 1, p. 311-321, 1984.

[24] VISWANATHAN, R.; JAYAS, D. S.; HULASARE, R. B. Sorption isotherms of tomato slices and onion shreds. Biosystems Eng., v. 86, n. 4, p. 465-472, 2003.

[25] ZHANG, X.W.; LIU, X.; GU, D.X.; ZHOU, W.; WANG, R.L.; LIU, P. Desorption isotherms of some vegetables. J. Sc. Food Agr., v. 70, p. 303-306, 1996.

\section{6 - AGRADECIMENTOS}

Os autores agradecem à Coordenação de Aperfeiçoamento de Pessoal de Nível Superior (Capes), pelo suporte financeiro. 